supplement of GIS lab exercises provide useful pedagogical tools. For a more substantively focused class on the spatial aspects of public health research, the book would serve as a good companion to a more topically-oriented text or compilation of articles.

One concern that I have about the book is that important geographic concepts are often buried in chapters. For example, the modifiable areal unit problem (MAUP) on pages $128-133$ is a subsection of chapter 4, "Mapping Health Information," and almost exclusively discusses the problem in terms of map-making. Given how students often come to GIS, I worry that this foundational concept would appear to them to be one about making maps, not about potential problems underlying areal data and their analysis. Burying the topic in a subsection within a chapter on mapping makes referencing the problem later difficult, as Cromley and McLafferty do in the final chapter when discussing neighborhood definitions in health disparities research. With the advantages of both hindsight and not being required me to implement the suggestion, I might have included a chapter on "geographical concepts" that collected topics like the MAUP, spatial reliability and validity of data, and hypothesis generation in a spatial context. All of the information exists in the book as written, it is just scattered throughout in a way that might make it difficult for students to grasp the generality of the concepts.

This relatively minor quibble does not dampen my enthusiastic recommendation of GIS and Public Health to students, professors, and practitioners alike. As GIS becomes an increasingly popular and useful tool for public health research and practice in the United States and abroad this comprehensive but accessible text should-and I expect will-continue to be a mainstay of GIS education of public health researchers and practitioners.

The next student knocking my door asking to learn GIS will certainly find herself reading it.

Michael D. M. Bader

Assistant Professor of Sociology and Faculty Affiliate of the Center on Health, Risk, and Society, American University.

\title{
Visualizing Data Patterns with Micromaps
}

By Daniel B. Carr and Linda Williams Pickle. 2010. Series: Interdisciplinary Statistical Series. Boca Raton, Florida: CRC Press (a Chapman and Hall book). $164+$ xvii.

ISBN 978-1-4200-7573-1, \$69.95

Micromaps are graphics that link statistical information to an organized set of small maps that can 'highlight geographic patterns and associations' among variables and facilitate hypothesis generation 'about plausible causes of the observed trends and relationships' (p.1). For almost two decades Daniel Carr and Linda Williams Pickle have been at the forefront in developing data visualization techniques, and their tour of micromaps is worth taking. The tour is accomplished in a short book containing seven chapters and an appendix of data sources and notes for the empirical examples visited on route. The examples focus on cancer and health service utilization but the diverse set includes studies of poverty, crime, education, and pollution as well as use of data on baseball performance, precipitation and growing season, and mammal brain sizes. The tour includes a self-paced element as the book is accompanied by a limited but evolving set of web resources providing access to micromap software, R-code, tutorials, and sample boundary files and data. Some of these additional resources allow users to 
incorporate their own data.

The tour begins with three chapters that provide necessary background for understanding micromaps and help the reader appreciate the intellectual transdisciplinary foundations on which micromaps are based. The authors 'primarily see adding micromaps as a way to enrich existing courses (p.xiii)' and these opening chapters help achieve that goal. Chapter 1 introduces row-labeled plots (a precursor to linked micromaps) and in an effective example walks the reader through simple design features that create perceptual groupings within a data set that can enhance data presentation, hypothesis generation, and data interpretation. The opening chapter also provides a brief overview of linked, conditioned, and comparative micromaps that are explored in greater depth in the second half of the book. At just 16 pages Chapter 2 provides a useful summary of 'research influencing micromap design' including the influence of among others Tukey, Cleveland, and Tufte in statistical graphics and cognition, MacEachren and Brewer in cartography, and Kosslyn in cognitive neuroscience. Coincidently perhaps Chapter 2 is the only chapter to include a 'further reading' section; in this instance providing to those wishing to delve a little deeper a short reference list on theoretical background and application of visualization theory. Chapter 3 summarizes well known techniques for 'enabling accurate comparisons' in complex data (including non-spatial) motivated by simplicity in design and appearance a la Tufte. The chapter closes with a section on engaging the analyst, essentially the requirement that micromap software incorporate 'tools and choices' that promote userinteraction and supporting materials for the analyst (e.g., help screens and tutorials). The fit of this latter section with the rest of the chapter is awkward unless read as the justification for the designed features of the Linked Micromap software (http://gis.cancer.gov/tools/ micromaps/) and its implementation by the State Cancer Profiles web-based system (http://statecancerprofiles.cancer. gov).

Chapter 4 builds off Chapter 3 providing a more detailed look at linked micromaps and specific design components. Starting with overall page layout the chapter describes examples of data encoding such as the use of bars, dots, lines and arrows in micromap graphics as well as the more incorporation of box plots, confidence intervals, and use of visual smoothing techniques. The chapter closes with the first, and quite brief, discussion of the use and availability of micromap software.

The second half of the micromap tour is worth the wait as the level of sophistication in micromaps is raised, and here the material is tied directly to the available software. Conditioned micromaps (Chapter 5) 'define subsets of geographic regions based on a crosscategorization using the conditioning variables that describe the regions' (p.81) and allow the viewer to explore hypotheses and to think spatially about the relationships between an outcome map of interest and potential risk factors. Several new design features are discussed drawing on the authors' software CCmaps which provides 'tools and choices' via the use of sliderbars, statistical summaries, and graphical plots (e.g., conditioned QQ plots). Comparative micromaps (Chapter 7), the newest form of micromap, introduce the time dimension allowing for studies of change over time as well as comparisons between subsets of data. The chapter introduces TCmaps software and in part because this is a prototype micromap there is emphasis on the appropriate use of comparative micromaps.

Micromaps have been used by cancer epidemiologists and cancer control researchers but can be effective tools in the social and behavioral sciences, including demography. This is ably demonstrated in an interesting final chapter titled 'putting it all together' (which does just that). Here the authors illustrate how micromap techniques can be used to examine and provide new insight regarding population change in Louisiana parishes prior to and after the 2005 hurricane season.

I enjoyed reading the book a great deal but I have some gripes. First, while I appreciate the history behind developments that lead to micromaps, the 
reference to, and the linkage with, other forms of geovisualization and exploratory spatial data analysis as well as to emergent trends in local analysis (spatial and non-spatial) was thin, and disappointingly so. Second, for the most part the book is well written but it includes several features that are uneven across chapters. Here I include the use of further reading suggestions (Chapter 2 only) and the use of a small number of 'grey boxes' containing summaries of research and/or key issues (Chapter 5 and 6 only). Why these boxed features were worth emphasizing over other topics/readings was not clear. Third, there are several websites referred to in the text and mention of several software packages. These could easily have been collated and included in an additional appendix. Finally, it is fair game to criticize the accompanying website as it is tightly coupled with the book. It is clearly a website in development' and other than a link to the authors' software is not as extensive or as dynamic (changing) as might be expected. This may all change if the micromap user-community grows, members contribute sample datasets and guides, and collectively they stimulate further enhancements in the interactive toolkit. I hope all this happens.

\section{Stephen A. Matthews}

Associate Professor of Sociology, Anthropology and Demography (Courtesy Geography), The Pennsylvania State University

\section{Introduction to Spatial Econometrics}

By James LeSage and R. Kelly Pace. 2009. Series: Statistics: Textbooks and Monographs. Boca Raton, Florida: CRC Press (a Chapman and Hall book). $354+$ xiii. ISBN 978-1-42006424-7, \$89.95

While spatial econometrics has been a primary analytic tool for spatial demographers, the computational and theoretical developments of the past quarter-century (since Anselin's seminal work (1988)) have not been thoroughly documented. LeSage and Pace admirably fill this void. They summarize a variety of innovative spatial regression models and elaborate on the relationships to traditional analytic perspectives. Throughout the text, the authors emphasize how to correctly interpret the estimated coefficients of spatial econometric models and provide empirical and experimental illustrations for the reader to follow. With the proliferation of spatial data and the resurgence of macro/spatial demography (Voss, 2007), I believe this book will become a necessary addition to the bookshelves of faculty and graduate students interested in spatial methods. Below I identify and summarize three reasons why LeSage and Pace's book will prove valuable to the field.

First, the breadth and depth of the book is appropriate for both beginners and advanced researchers. The topics span the traditional spatial regression models (Chapters 2 and 3), such as spatial autoregressive model (SAR), spatial error model (SEM), and spatial Durbin model (SDM), to the latest developments in spatial modeling (Chapters 9 and 10) such as matrix exponential spatial specification (MESS). Although these models seem unrelated, the authors explain how the recent methodological developments are derived from traditional models. As for model estimation, the maximum likelihood (ML) approach is introduced (Chapter 3), but the authors apply the Markov chain Monte Carlo (MCMC) and Bayesian perspective to spatial econometrics (Chapter 5). In Chapters 6, 7, and 8, both MCMC and ML estimation approaches are further explained and used in different analytic settings and model selections. The advantages and drawbacks of these approaches are demonstrated with empirical examples. The combination of the detailed discussions of fundamental concepts and the 